\title{
Distinct roles of two alternative splice variants of matrilin-2 in protein oligomerization and proteolysis
}

\author{
LONGXUAN LI $^{1 *}$, LIANGQING ZHANG ${ }^{2 *}$, YIMING SHAO $^{1 *}$, GUIRONG WANG $^{3}$, RUJUN GONG $^{4}$, \\ ZHENGKE WANG ${ }^{5}$, JINWU PENG ${ }^{6}$, SHUHUA WANG $^{7}$, DAVID GENOCHIO $^{8}$, BIN ZHAO $^{1}$ and JUNMING LUO ${ }^{1,8}$

\begin{abstract}
Departments of ${ }^{1}$ Neurology and ${ }^{2}$ Anesthesiology, Guangdong Medical College Affiliated Hospital, Zhanjiang 524001, P.R. China; ${ }^{3}$ Department of Surgery, SUNY Upstate Medical University, Syracuse, NY; Departments of ${ }^{4}$ Internal Medicine and ${ }^{5}$ Orthopaedic Research, Brown Medical School/Rhode Island Hospital, Providence, RI, USA; ${ }^{6}$ Department of Pathology, Xiangya Medical School, Central South University, Changsha 410073, Hunan, P.R. China; ${ }^{7}$ Kidney Institute, Department of Medicine, University of Kansas; ${ }^{8}$ VA Medical Center at Kansas City Missouri, Kansas City, MO, USA
\end{abstract}

Received June 10, 2012; Accepted August 20, 2012

DOI: $10.3892 / \mathrm{mmr} .2012 .1056$

\begin{abstract}
Matrilin-2 (matn2) contains a unique domain, between the second von Willebrand factor A (vWFA) domain and the C-terminal coiled-coil domain, with no sequence homology with other family members. Complementary DNA (cDNA) sequence analysis of matn2 expression in both mice and humans revealed an alternative splice site in the region of the unique domain, which forms a short and a long splicing variant (containing an additional 19 amino acids). However, the expression heterogeneity of the alternative spliced variants, and the roles of the unique domain in oligomerization and proteolysis of matn 2 are unknown. In this study, we examined the expression of the two alternative splice variants of matn2 in several skeletal and non-skeletal tissues by reverse transcription-polymerase chain reaction. Both splice variants of matn2 were detected at the mRNA level in all tissues studied. To explore the biochemical significance, several minigene constructs containing the second vWFA domain, the unique domain (with either a long or short form) and the coiled-coil domain of mouse mini matn2 were generated. Ectopic expression of these constructs demonstrated that the long form of matn2 is capable of self-assembling into several oligomeric forms, including a tetramer, trimer, pentamer or multimer; but the short form is only capable of forming a tetramer, trimer or dimer. Moreover, we observed that the splice variants of
\end{abstract}

Correspondence to: Professor Junming Luo or Professor Bin Zhao, Department of Neurology, Guangdong Medical College Affiliated Hospital, 57 South Renmin Road, Zhanjiang 524001, Guangdong, P.R. China

E-mail: jluo099@yahoo.com

E-mail: Zhaobine@163.net

*Contributed equally

Key words: Matrilin-2, alternative splicing, oligomerization, proteolysis, posttranslational modification matn 2 are important in modulating matn2 cleavage when co-expressed with matrilin-1 or matrilin-3. These results indicate that the two alternative splice variants have distinct roles in the processes of post-translational modification of matn2, which may have an impact on the homeostasis of the matrilin filamentous network of the extracellular matrix.

\section{Introduction}

Matrilin-2 (matn2) is the largest member of the matrilin family, with multiple domains of extracellular matrix protein, and plays a role in the formation of filamentous network structures and connects different molecules, such as collagens, other matrilins, aggrecan, small leucine-rich proteoglycans or cartilage oligomeric matrix protein (COMP), to form the extracellular macromolecular network with adaptor functions (1-3). Based on the analysis of the mouse matn2 sequence, the precursor containing 956 amino acids consists of an N-terminal putative signal peptide, two von Willebrand factor type A-like domains (vWFA) connected by 10 epidermal growth factor-like (EGF) repeats, one unique domain and a series of heptad repeats at its C-terminal coiledcoil domain; the predicted molecular mass is approximately $106.8 \mathrm{kDa}(2-3)$. The vWFA domain is one of the ubiquitously distributed structural modules. There are seven vWFA domains in all four members of the matrilin family. The vWFA domain-containing molecules have been identified ubiquitously in the extracellular matrix, cell membrane and nucleus, and they participate in cell adhesion, protein-protein interaction and the formation of multiprotein complexes (4-5). The EGF domain is another ubiquitously distributed structural module consisting of 30 to 40 amino acid residues (5-7). The main structure is a two-stranded $\beta$-sheet followed by a loop to a C-terminal short two-stranded sheet. The EGF domain has six evolutionarily conserved cysteine residues, which indicates that this domain is involved in forming disulfide bonds. The EGF domain proteins include many extracellular matrix, membrane and signal pathway proteins (5-7). Multiple functions of both vWFA and EGF domains of matrilins have 
Table I. Primers used in this study.

\begin{tabular}{rll}
\hline Primers & \multicolumn{1}{c}{ Primer sequences (5'-3') } & \multicolumn{1}{c}{ PCR purpose } \\
\hline 1 & TAA TAC GAC CTA TAG GG & T7, amplifying inserts from pCDNA3.1 \\
2 & CTA GAA GGC AAC AGT CGA GG & BGH, amplifying inserts from pCDNA3.1 \\
3 & CGC GGC CGC ATG GAC GGG ATT TTC TGC & Clone matn2 \\
4 & GGC TCG AGT CTG TAT TTT AGG CGA TT & Clone matn2 \\
5 & GGT CCT GGC TTT CAC AGA TGC CTT CTT & Delete unique region \\
6 & CAT CTG TGA AAG CCA GGA CCA ATG CAA & Delete unique region \\
7 & GAT GCA CTG AAG GCC CAA TTG AC & Amplifying 57 bp splicing, 1st PCR \\
8 & TGA GTC ATT TCT TCT AAG CGC TGG G & Amplifying 57 bp splicing, 1st PCR \\
9 & TGC CTC TGA GCC CAT TGA CAA G & Amplifying 57 bp splicing, 2nd PCR \\
10 & TTT GCA TTG GTC CTG GCT TTC TT & Amplifying 57 bp splicing, 2nd PCR \\
11 & GCA TCT TTT GTC ATC ATC GTC CTT & Add FLAG tag \\
12 & GAT GAC AAA AAG AGA TGC ACT GAA GGC & Add FLAG tag \\
\hline
\end{tabular}

been extensively investigated (8-11). The coiled-coil domain at the $\mathrm{C}$-terminus is a nucleation site for the potential homoand hetero-oligomerization.

Matn2 contains a unique domain without any sequence homology to other known molecules (1-3). The unique domain is located between the second vWFA domain and the coiled-coil domain (1-3). In mouse matn2, the domain consists of 75 amino acid residues $(1-2,11)$. Sequence analysis of expressed complementary DNA (cDNA) indicated that the unique domain of either mouse or human matn2 has 19 triplets alternatively retained or spliced out $(1,2,11,12)$. While the function of the other domains of matn2 including vWFA, EGF and coiled-coil has been extensively studied, the biological role of the unique domain is unclear. In this study, we examined the alternative splicing expression of matn 2 in several tissues from newborn mice, and then explored the biochemical properties of protein oligomerization and proteolysis by ectopic expression, in which the recombinant construct expressed either the long or short alternative splice form of the unique domain of matn2.

\section{Materials and methods}

RNA extract from animal tissues, and reverse transcription polymerase chain reaction (RT-PCR). The various skeletal tissues, including skull, knee joint, rib, spinal column, and several non-skeletal tissues, such as kidney, brain, skin, ear, stomach and colon, were harvested from newborn C57BL/6 mice. Tissue weighing approximately $20 \mathrm{mg}$ was homogenized, and total RNA was isolated using an RNeasy Kit (Qiagen, Valencia, CA, USA). First-strand cDNA was synthesized using Superscript II kit (Invitrogen, Carlsbad, CA, USA) according to the manufacturer's instructions. To ensure the sensitivity, nested PCR was performed using first the distal primers and then the proximal primers (Table I) for mouse matn2. To avoid false results, mini matn 2 with and without 57-bp splicing plasmids was used as a positive control and water was used to replace total RNA as a negative control. The study was approved by the ethics committee of Guangdong Medical College, Zhanjiang, China.
Preparation of mini matrilin gene constructs. Mini matn2 cDNA fragments were cloned from the newborn C57BL/6 mouse rib library. Suitable primers (Table I) introduced a 5'-terminal NotI and a 3'-terminal XhoI restriction enzyme site into the expression vector pcDNA3.1/v5-His (Invitrogen). To ensure the secretion from cells, a signal peptide cDNA sequence of matrilin-1 (matn1) was added into the 5'-terminal of the matn 2 cDNA fragment $(13,14)$. To study the function of the unique domain, a genetically engineered deletion of the unique domain mutant of mini matn2 was linked by overlapping PCR with the described primer sets (Table I). To examine the interaction between matn 2 and matn 1 or matrilin-3 (matn3), the whole length of matn1 and mini matn3 constructs from previous experiments were used in this study $(13,14)$. In addition, a genetically engineered FLAG tag was introduced into all the mini matn 2 cDNA constructs, which allowed the identification of the recombinant protein. Therefore, all the matn 2 constructs contained the FLAG tag and the V5 tag, but the matn 1 and matn 3 constructs only contained the V5 tag (Fig. 1). The sequences of the recombinants were confirmed by DNA sequencing.

Transfection of plasmid DNA. Plasmids containing mini matn2, matn1 and mini matn 3 were transfected or co-transfected into COS-1 cells using Lipofectamine 2000 (Life Technologies, Invitrogen) according to manufacturer's instructions. Briefly, COS-1 cells were trypsinized and counted. Each $60 \mathrm{~mm}$ plate was seeded with $6 \times 10^{5}$ cells overnight, and reached $70 \%$ confluence in DMEM supplied with $10 \%$ FBS (Life Technologies). On the following day, the cells were rinsed with DMEM three times, and then transfected/ co-transfected with plasmids/Lipofectamine $2000 \mathrm{mix}$ as described previously $(13,14)$. For a single transfection $4.5 \mu \mathrm{g}$ of plasmid was used, and $3 \mu \mathrm{g}$ of each plasmid was used when two plasmids were co-transfected. Seventy-two hours after transfection, the conditioned media was collected for western blotting analysis $(13,14)$.

SDS-polyacrylamide gel electrophoresis and western blotting. Conditioned media was mixed with $2 \mathrm{X}$ SDS reduced or 
A
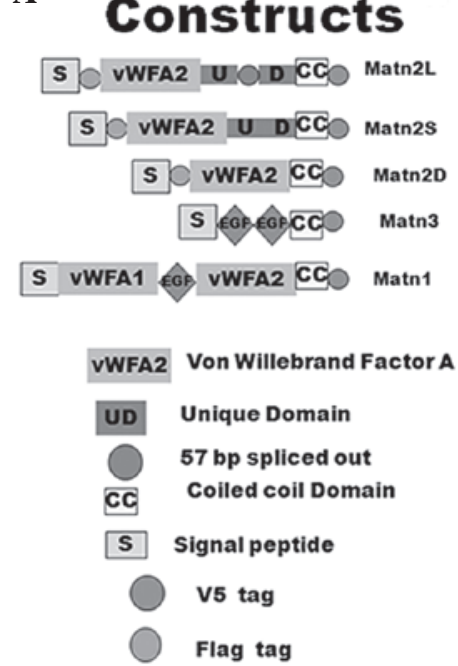

B

\section{Western Blot}
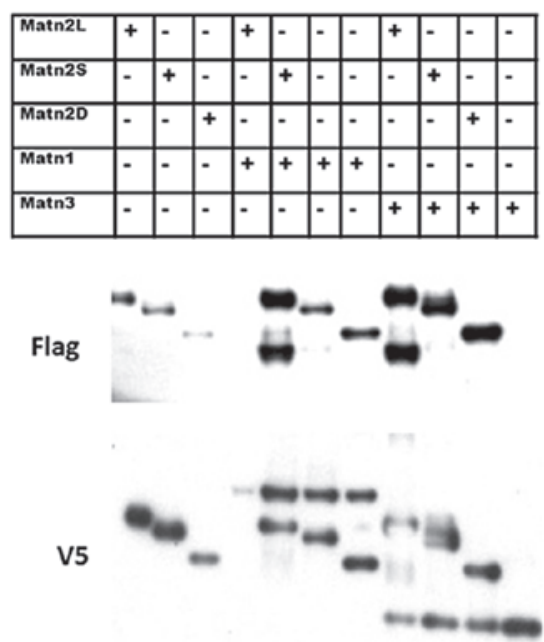

Figure 1. (A) Schematic drawings of the domain structure of recombinant matrilin variants and (B) analysis of recombinant matrilin products. The recombinant proteins of matn 2 single transfection and matn 2 co-transfection with matn 1 or matn 3 were separated on a $4-15 \%$ gel, blotted to a membrane and incubated with antiserum FLAG tag (B, upper panel, only detecting recombinant matn2 product), and incubated with antiserum V5 tag (B, lower panel, detecting all matrilin products) under reducing conditions.

non-reduced gel loading buffer. The reduced loading buffer contained $5 \% \beta$-mercaptoethanol. After boiling for $10 \mathrm{~min}$, samples were loaded onto 4-15\% gradient gels (Bio-Rad, Hercules, CA, USA). After electrophoresis, proteins were transferred onto immobilon-polyvinylidene difluoride membrane (Millipore, Billerica, MA, USA). The blots were blocked with 5\% non-fat milk (Bio-Rad). The monoclonal antibody against V5 tag (Invitrogen, diluted 1:5000) and the polyclonal antibody against FLAG tag (Affinity BioReagents, Pierce, Rockford, IL, USA; diluted 1:1000) were used as the primary antibodies, respectively. The secondary antibodies were horseradish peroxidase-conjugated goat anti-mouse or goat anti-rabbit IgG $(\mathrm{H}+\mathrm{L})$ (Bio-Rad, diluted 1:5000). Visualization of immunoreactive proteins was achieved using the ECL Western blotting detection reagents (Amersham Pharmacia Biotech, Amersham, UK) and then by exposing the membrane to Kodak X-Omat AR film. The molecular weights of the immunoreactive proteins were determined with two sets of protein markers (13).

\section{Results}

Tissue distribution of mouse matn2 splice variants. To examine expression of matn2 splice variants, forward and reverse primers located upstream and downstream of the splicing region in the unique domain were used. RT-PCR products of long and short splice variants were matn $2 \mathrm{~L}$ (337 bp) and matn2S (280 bp), respectively. The long and short splice variants of matn 2 at the mRNA level were observed in all the mouse skeletal and non-skeletal tissues examined (Fig. 2). The skeletal tissues included knee, sterna, skull, rib and backbone, and non-skeletal tissues included kidney, skin, heart, stomach, small and large intestine. The results indicated that the long and short splicing variants are expressed in all examined tissues, and may be important in the extracellular matrix network.

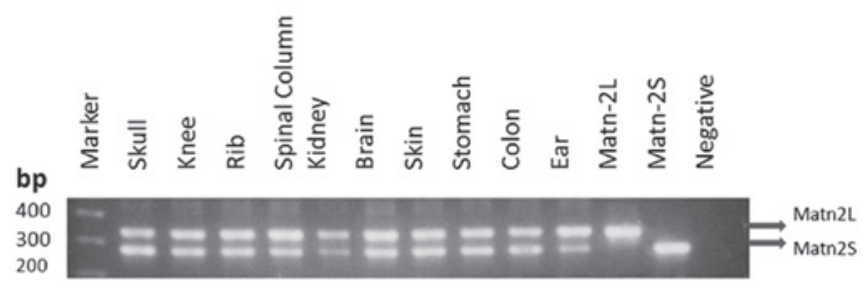

Figure 2. Differential expression of the variable unique region of matrilin-2 was assessed in newborn mouse tissues by RT-PCR. RT-PCR yielded two bands corresponding to matn2L (337 bp) and matn2S (280 bp), respectively. Both splice variants of unique domain of matrilin- 2 mRNA were observed in all the mouse tissues examined.

Alternative splicing variants affect homo-oligomerization. Matn2L and matn2S were cloned into the expression vector and matn2L and matn2S constructs were generated. Matn2L contained the entire unique domain of matn2, while matn2S lacked the 57-bp sequence that encodes 19 amino acid residues in the unique domain (Fig. 1A). A FLAG tag was ligated to the N-terminus, and a V5 tag and 6 His tags were fused into the $\mathrm{C}$-terminus of the minigene. The recombinant protein was identified with antibody against these tags (Fig. 1A). To express these recombinant splicing variants, the constructs with either matn2L or matn2S were transfected into COS-1 cells. The secreted protein products in the conditioned medium were examined by a western blotting analysis of the FLAG tag (Fig. 1B, upper panel) and V5 Tag (Fig. 1B, lower panel), respectively.

The long form of matrilin-2 (matn2L), under non-reduced conditions, showed 4 bands: a dominant band at $156 \mathrm{kDa}$, consisting of the matn2L tetramer (see Table II); a weak band at $117 \mathrm{kDa}$ (trimer); a faint band at $196 \mathrm{kDa}$ (pentamer); and a multimer (Fig. 3A, lane 1; and 3B, upper panel, lane 1). In the reduced gel, only one band at approximately $39 \mathrm{kDa}$ (monomer) was detected (Fig. 3B, lower panel, lane 1). Under 
Table II. Calculated molecular weight of matn1, -2 and -3 homo- and hetero-oligomers.

\begin{tabular}{lcccl}
\hline & $\begin{array}{c}\text { Monomer } \\
\mathrm{kDa}\end{array}$ & $\begin{array}{c}\text { Dimer } \\
\mathrm{kDa}\end{array}$ & $\begin{array}{c}\text { Trimer } \\
\mathrm{kDa}\end{array}$ & $\begin{array}{c}\text { Tetramer } \\
\mathrm{kDa}\end{array}$ \\
\hline Matn2L & 39 & 78 & 117 & $156(\mathrm{M} 2) 4$ \\
& & & & $188(\mathrm{M} 2) 2(\mathrm{M} 1) 2$ \\
& & & & $204(\mathrm{M} 2) 1(\mathrm{M} 1) 3$ \\
& & & & $137(\mathrm{M} 2) 3(\mathrm{M} 3) 1$ \\
Matn2S & 37 & 74 & 111 & $148(\mathrm{M} 2) 4$ \\
& & & & $184(\mathrm{M} 2) 2(\mathrm{M} 1) 2$ \\
& & & & $202(\mathrm{M} 2) 1(\mathrm{M} 1) 3$ \\
& & & & $131(\mathrm{M} 2) 3(\mathrm{M} 3) 1$ \\
$124(\mathrm{M} 2) 4$ \\
Matn2D & 32 & 64 & 96 & $172(\mathrm{M} 2) 2(\mathrm{M} 1) 2$ \\
& & & & $199(\mathrm{M} 2) 1(\mathrm{M} 1) 3$ \\
& & & & $102(\mathrm{M} 2) 3(\mathrm{M} 3) 1$ \\
Matn1 & 55 & & & \\
Matn3 & 20 & & & \\
\hline
\end{tabular}

Matn2L, matrilin-2 long; Matn2S, matrilin-2 short; Matn2D, matrilin-2 deletion; Matn1, matrilin-1; Matn3, matrilin-3.

non-reduced conditions, the short form (matn $2 \mathrm{~S}$ ) exhibited 3 bands: a predominant band at $148 \mathrm{kDa}$ (tetramer); a band at $111 \mathrm{kDa}$ (trimer), a weak band with $74 \mathrm{kDa}$ (dimer) (Fig. 3A, lane 3 , and $3 \mathrm{~B}$, lane 2 ). Only a $37-\mathrm{kDa}$ (monomer) band was observed under the reduced conditions (Fig. 3B, lower panel, lane 2). To further determine the role of the unique domain of matn2 in the oligomerization, a deletion mutant construct (matn2D) was generated and expressed the mutated protein in the COS-1 cells. Only a band of approximately $124 \mathrm{kDa}$ (tetramer) was observed (Fig. 3A, lane 5, and 3B, upper panel, lane 3) on the blot under non-reduced conditions. As expected, there was a $32-\mathrm{kD}$ band as a monomer of the mutant matn2D on the reduced blot (Fig. 3B, lower panel, lane 3). The results demonstrated that the long form (matn2L) produced different oligomeric forms from the short form (matn2S) and suggested that the alternative splicing mechanism in matn 2 expression plays a role in modulating stochiometry of the matn 2 oligomerization. These different oligomeric forms, matn2L and matn2S, may play different roles in the filamentous network.

Splice variants of matn2 do not affect hetero-oligomerization with matn1 and matn3. To examine the heterogenic oligomerization of matn2 with matn1 and 3, each of the mini matn2 constructs (matn2L and matn2S) was co-transfected with matn1 and mini matn3. When matn2L was co-transfected with matn1 (Fig. 3A, upper panel) and blotted with antiserum against FLAG tag, 2 additional bands (approximately 188 and $204 \mathrm{kDa}$ ) were found higher than the homotetramer (Fig. 3A, lane 2), compared with the single construct transfection (Fig. 3A, lane 1). According to the molecular weight calculations (Table II), the major 188-kDa product was a form of (Matn1)2(Matn2L)2 hetero-oligomer, and the weak band (approximately $204 \mathrm{kDa}$ ) was a form of (Matn1)3(Matn2L)1 hetero-oligomer (Fig. 3A, upper panel, lane 2). When matn $2 \mathrm{~S}$ was co-transfected with matn1 (Fig. 3A), there were also 2 additional bands observed, whose sizes (approximately 184 and $202 \mathrm{kDa}$ ) (Fig. 3A, lane 4) were larger than the homotetramer products shown in the
A
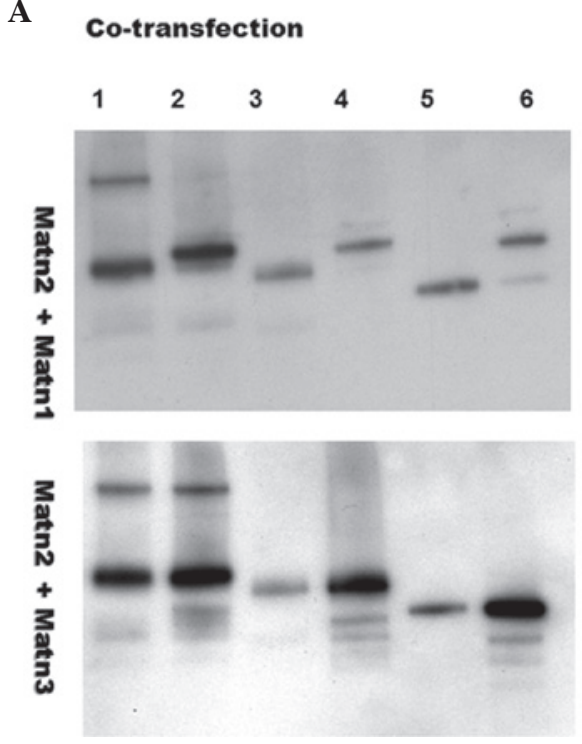

B

Single Transfection

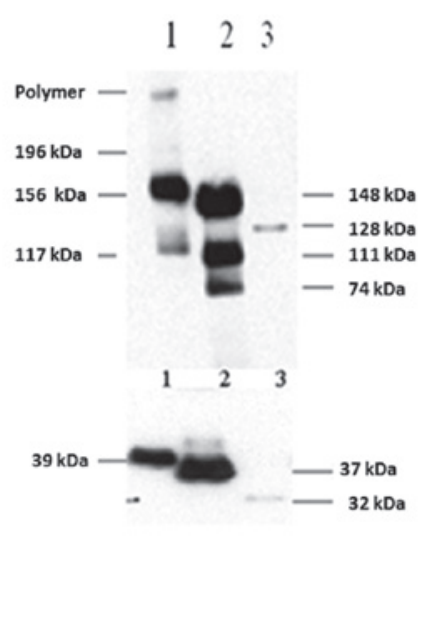

Figure 3. Co-transfection of matn2 with matn1 (Fig. 3A, upper panel) and matn3 (Fig. 3A, lower panel) and single transfection matn2 (Fig. 3B). Co-transfection matn2 with matn1 (Fig. 3A, upper panel), side by side comparison of matn2 single transfection (lane 1,3 and 5) with matn2 co-transfection with matn1 (lane 2, 4 and 6). Two heterotetramers of (Matn1)2(Matn2)2 and (Matn1)3(Matn2)1 were found. Co-transfection of matn2 with matn3 (Fig. 3A, lower panel), side by side comparison of matn2 single transfection (lane 1,3 and 5) with matn2 co-transfection with mini matn3 (lane 2, 4 and 6). One heterotetramer of (Matn2L)3(Matn3)1 was found. In the single transfection under non-reduced conditions (Fig. 3B, upper panel), lane 1 (matn2L) contained 4 tetramer products at $156 \mathrm{kDa}$, a trimer at $117 \mathrm{kDa}$, a pentamer at $196 \mathrm{kDa}$ and a higher order multimer; lane 2 (matn2S) contained 3 tetramer products at $148 \mathrm{kDa}$, a trimer at $111 \mathrm{kDa}$ and a dimer at $74 \mathrm{kDa}$; lane3 (matn2D) contained only a tetramer at $124 \mathrm{kDa}$. Upon reduction, all of these bands were reduced to $39 \mathrm{kDa}$ (matn2L), $37 \mathrm{kDa}($ matn2S) and $32 \mathrm{kDa}$ of the monomer (lower panel). 


\begin{tabular}{|c|c|c|c|c|c|c|c|c|}
\hline & 1 & 2 & 3 & 4 & 5 & 6 & 7 & 8 \\
\hline Matn2L & - & + & - & - & + & - & - & - \\
\hline Matn2S & - & - & + & - & - & + & - & - \\
\hline Matn2D & - & - & - & + & - & - & + & - \\
\hline Matn1 & + & + & + & + & - & - & - & - \\
\hline Matn3 & - & 1- & - & - & + & + & + & + \\
\hline
\end{tabular}

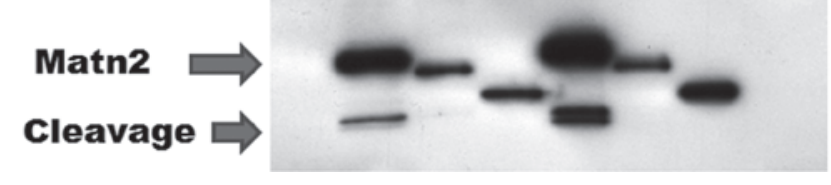

Figure 4. Analysis of matn2 cleavage products. Using SDS-PAGE analysis, matn 2 co-transfections with matn1 or matn3 were separated on a 4-15\% gel, blotted to a membrane and incubated with antiserum for FLAG tag. There were no cleavage products in the single transfection with matn1 (lane 1) and matn3 (lane 8). After matn2L co-transfection with matn1, there was a strong band at approximately $26 \mathrm{kDa}$, and a faint $24-\mathrm{kDa}$ product (lane 2) For matn2L co-transfection with matn3, those two products were almost equal (lane 5). In matn2S, there was a very faint $24-\mathrm{kDa}$ product in the co-transfection with matn1 (lane 3), but it was difficult to detect this faint band when matn $2 \mathrm{~S}$ was co-transfected with matn3 (lane 6). However, when the unique region was deleted, there were no cleavage products in matn $2 \mathrm{D}$ (lanes 4 and 7).

single construct transfection (Fig. 3A, lane 3). The 184-kDa form, (Matn1)2(Matn2S)2, was a prominent product, and the 202-kDa form, (Matn1)3(Matn2S)1, was a less prominent product of the hetero-oligomer. Furthermore, when the matn2D mutant was co-transfected with matn1 (Fig. 3A), there were two bands higher than the homotetramer, and the other band was lower than the homotetramer (Fig. 3A, lane 6) when compared with single transfection (Fig. 3A, lane 5). The dominant product (approximately $172 \mathrm{kDa}$ ) was a hetero-oligomeric form of (Matn1)2(Matn2D)2, the band with $199 \mathrm{kDa}$ was a (Matn1)3(Matn2D)1 hetero-oligomer and the $96 \mathrm{kDa}$ product was a trimeric form of matn2D (Fig. 3A, lane 6).

When matn2L was co-transfected with matn3 (Fig. 3A, lower panel), under non-reducing conditions and detected with FLAG tag, there was a weak band (approximately $137 \mathrm{kDa}$ ) between the homotetramer and homotrimer (Fig. 3A lower panel, lane 2), compared with single transfection (Fig. 3A lower panel, lane 1). The product was believed to be a heterotetrameric form of (Matn2L)3(Matn3)1 (Fig. 3A, lower panel, lane 2). When matn2S was co-transfected with matn3 (Fig. 3A, lower panel, lane 4), there was also an additional band $(131 \mathrm{kDa})$ between the homotetramer and homotrimer, which was believed to be a (Matn2S)3(Matn3)1 hetero-oligomer (Fig. 3A, lower panel, lane 4). Matn2D was co-transfected with matn3 (Fig. 3A, lower panel), and 3 additional bands were observed that were smaller than the homotetramer (Fig. 3A, lower panel, lane 6). They were thought to represent a $105-\mathrm{kDa}$ hetero-oligomer (Matn2D)3(Matn3)1, a $96 \mathrm{kDa}$ homotrimer and a $64-\mathrm{kDa}$ homodimer. These results indicated that the unique domain of matn 2 may not be important to assemble hetero-oligomers with matn1 and matn 3 in vitro.

Alternative splicing variants have an impact on proteolysis. Using SDS-PAGE analysis of the recombinant FLAG tag, there were two cleavage products when mini matn 2 was co-transfected with matn1 or mini matn3, but no cleavage product was observed in matn2 single transfection (Fig. 1B, upper panel). Those products were not recognized by V5 tag (Fig. 1B, lower panel). The molecular sizes of the products were approximately 26 and $24 \mathrm{kDa}$, respectively. When matn2L was co-transfected with matn1, there was a dominant cleavage product (approximately $26 \mathrm{kDa}$ ), and a very faint 24-kDa cleavage product (Fig. 4, lane 2). When matn2L was co-transfected with matn3, the density of two cleavage products was found to be almost equal (Fig. 4, lane 5). Furthermore, only a very faint $24-\mathrm{kDa}$ cleavage product was observed when matn2S was co-transfected with matn1 (Fig. 4, lane 3), but it was difficult to find this faint band when matn $2 \mathrm{~S}$ was co-transfected with matn3 (Fig. 4, lane 6). However, the unique region of the matn2 was deleted completely; no cleavage product was observed (Fig. 4, lanes 4 and 7). These results demonstrated that the unique domain of matn2 is critical for proteolysis and two alternative splicing variants of matn 2 have different abilities in the processes of post-translational modification.

\section{Discussion}

Matn2 is a novel family member of the filament-forming oligomeric adapter proteins that are involved in the development and homeostasis of the extracellular matrix network (1-3). At present, the information on the biological function of matn2 is limited. Matn2 is one of the major components of basement membranes and a putative adaptor molecule of the extracellular matrix, which can form both collagen-dependent and collagen-independent filamentous networks $(1-3,14,15)$, and has been involved in the reorganization of tissue architecture in liver cirrhosis and hepatocellular carcinoma (16), the balance of keratinocyte and fibroblasts in response to wounding (17), and participated in peripheral nerve regeneration (18), and is downregulated in the early phase of muscle injury and then increased in its late phase (19). Matn2 expression was elevated in the majority of cases of human sporadic pilocytic astrocyoma (20), liver cirrhosis and hepatocellular carcinoma (16). Seven putative Smad-binding sites exist within the human matn 2 promoter as well as exon I (17). Although the functions of this filamentous network adaptor of the matn 2 molecule are accumulating, gene-deficient mice show no gross abnormalities during embryonic or adult development, with a normal lifespan (15). Future studies with separated isoform gene-deficient mice may provide further information on the distinct roles of splicing variants of this molecule.

Matn2 is an inherent component of all types of dense and loose connective tissue, bone, cartilage, some epithelia and in a variety of organs $(1-3,15)$. In this study, we found that both long and short splice variants of the unique domain of matn 2 at the mRNA level were expressed in all the mouse tissues examined, including skeletal tissues such as knee, sterna, skull, rib and backbone, and non-skeletal tissues such as kidney, skin, heart, stomach, small and large intestine.

Matn2 is a multiple domain oligomeric molecule that is capable of self-assembly and co-assembly with a variety of other members of the family (1-3). The heptad repeat coiled-coil domain at the C-terminus is believed to respon- 
sible for the oligomerization of the molecule (6-7). Certain data showed that the second vWFA domain also plays a role in regulating matrilin oligomeric formation $(13,14,21)$. In the present study, we demonstrated that the splicing of the unique domain of matn 2 played an essential role in homo-oligomerization, but not hetero-oligomerization with other members. The short form of the splicing variant lacks 19 residues (EPEPVTIKIKDLLSCSNFA) in the unique region, which includes one cysteine. This cysteine may play a role in forming the covalent disulfide linkage responsible for pentamer and multimer homo-oligomerization. However, this cysteine appears to not be involved in the covalent disulfide bond formation between matn 2 and others (matn1, matn3), as both long and short variants have the same forms of hetero-oligimers. To further confirm the role of this cysteine in the oligomerization of matn2, it is necessary to mutate the cysteine and examine homo-oligomerization of the long and short splicing variants in future studies. In addition, the coiledcoil domain of matn 2 may be the only domain involved in the hetero-oligomerization.

Proteolysis is a major post-translational modification used to modify the function of proteins (22-24). Proteolysis is very complex. This complexity is three-fold. First, in the physiological condition, there are a mixture of different homo-oligomers and hetero-oligomers $(8,11,13,16,17)$. These oligomers are participants of the extracellular matrix network (1-3). Second, the oligomeric proteins have a bouquet-like structure and bind to a variety of different molecules $(11,22)$. Therefore, the avidity of their interactions is dependent on the number of subunits and domains present (22-24). The balance between the synthesis and degradation is important to homeostasis of the extracellular network (25). The adaptor functions of matrilins may be modulated by both physiological and pathophysiological proteolysis that causes the loss of single subunits and thereby decreases the binding avidity (22-24). Third, the cleavage occasionally releases protein fragments that have completely new functions (1-3). In this study, the splicing variants of the matn2 unique domain were shown to have a distinct role in protein cleavage, particularly when they are co-expressed with matn1 or matn3. Different activity was observed in the two splicing variants in in vitro experiments, indicating that the two splicing variants may have different biological functions. The splicing region in the unique domain may be important for matn2 to form a variety of oligomeric forms and to provide a functional diversity in the extracellular matrix proteins. In the future, investigating the proteolytic processing and the functions of cleavage products may provide some important information of the balance on matn2 filamentous network in the extracellular matrix.

In conclusion, the expression, splicing, assembly and proteolysis of extracellular matrix molecules are often determined by complex regulatory processes including transcriptional and translational events, as well as post-translational modifications. However, there are only a few studies stating that matn2 is involved in reorganization of tissue architecture of the extracellular matrix. In this study, we demonstrated that the splicing of the unique domain of matn 2 was observed in all mouse tissue examined, and played an essential role in regulating oligomerization and proteolysis.

\section{Acknowledgements}

The authors are grateful to Drs. Yue Zhang (Department of Orthopaedic Research, Pennsylvania State University) and Qian Chen (Department of Orthopaedic Research, Brown University) for providing matrilin-1 and mini matrilin-3 constructs. The authors also thank Drs. Mukut Sharma and Damaneep Walia (VA Medical Center at Kansas City Missouri) for their critical reading of this manuscript. This study was supported in part by the National Natural Science Foundation of China (No. 81171244), and by the Science and Technology Planning Project of Guangdong Province of China (No. 2011B031800110) and the Guangdong Natural Science Foundation of China (No. S2011010004095).

\section{References}

1. Klatt AR, Becker AA, Neacsu CD, Paulsson M and Wagener R: The matrilins: modulators of extracellular matrix assembly. Int J Biochem Cell Biol 43: 320-330, 2011.

2. Deak F, Piecha D, Barchrati C, Paulsson M and Kiss I: Primary structure and expression of matrilin-2, the closest relative of cartilage matrix protein within the von Willebrand factor type A-like module superfamily. J Biol Chem 272: 9268-9274, 1997.

3. Piecha D, Muatoglu S, Morgelin M, Hauser N, Studer D, Kiss I, Paulsson M and Deak F: Matrilin-2, a large, oliomeric protein, is expressed by a great variety of cells and forms fibrillar networks. J Biol Chem 274: 13353-13361, 1999.

4. Whittaker CA and Hynes RO: Distribution and evolution of von Willebrand/integrin A domains: widely dispersed domains with roles in cell adhesion and elsewhere. Mol Biol Cell 13: 3369-3387, 2002.

5. Luo J and Wan Y: Tightly regulated distribution of family members of proteins is related to social property in the open body system. Int J Mol Med 17: 411-418, 2006.

6. Wouters MA, Riquoutsos I, Chu CK, Feng LL, Sparrow DB and Dunwoodie SL: Evolution of distinct EGF domains with specific functions. Protein Sci 14: 1091-1103, 2005.

7. Rao Z, Handford P, Mayhew M, Knott V, Brownlee GG and Stuart D: The structure of Ca (+2)-binding epidermal growth factor-like domain: its role in protein-protein interactions. Cell 82: 131-141, 1995.

8. Pan $\mathrm{OH}$ and Beck $\mathrm{K}$ : The C-terminal domain of matrilin-2 assembles into a three-stranded alpha-helical coiled coil. J Biol Chem 273: 14205-14209, 1998.

9. Paulsson M, Piecha D, Segat D, Smyth N and Wagener R: The matrilins: a growing family of A-domain-containing protein. Biochem Soc Trans 27: 824-826, 1999.

10. Frank S, Schulthess T, Landwehr R, Lustig A, Mini T, Jeno P, Engel J and Kammerer RA: Characterization of the matrilin coiled-coil domains reveals seven novel isoforms. J Biol Chem 277: 19071-19079, 2002.

11. Piecha D, Wiberg C, Morgelin M, Reinhardt DP and Deak F. Matrilin-2 interacts with itself and with other extracellular matrix proteins. Biochem J 367: 715-721, 2002.

12. Muratoglu S, Krysan K, Balazs M, Sheng H, Zakany R, Modis L, Kiss I and Deak F: Primary structure of human matrilin-2, chromosome location of the MATN2 gene and conservation of an AT-AC intron in matrilin genes. Cytogenet Cell Genet 90: 323-327, 2000.

13. Zhang Y and Chen Q: Changes of matrilin forms during endochondral ossification. Molecular basis of oligomeric assembly. J Biol Chem 275: 32628-32634, 2000.

14. Chen Q, Zhang Y, Johnson DM and Goetinck PF: Assembly of a novel cartilage matrix protein filamentous network: molecular basis of differential requirement of von Willebrand factor A domains. Mol Biol Cell 10: 2149-21462, 1999.

15. Mates L, Nicolae C, Morgelin M, Deak F, Kiss I and Aszodi A: Mice lacking the extracellular matrix adaptor protein matrilin-2 develop without obvious abnormalities. Matrix Biol 23: 195-204, 2004.

16. Szabo E, Korpos E, Batmunkh E, Lotz G, Holczbauer A, Kovalszky H, Deak F, Kiss I, Schaff Z and Kiss A: Expression of matrilin-2 in liver cirrhosis and hepatocellular carcinoma. Pathol Oncol Res 14: 15-22, 2008. 
17. Ichikawa T, Suenaga Y, Koda T, Ozaki T and Nakagawara A: $\Delta$ Np63/BMP-7-dependent expression of matrilin-2 is involved in keratinocyte migration in response to wounding. Biochem Biophys Res Comm 369: 994-1000, 2008.

18. Malin D, Sonnenberg-Riethmacher E, Guseva D, Wagener R and Aszodi A: The extracellular-matrix protein matrilin 2 participates in peripheral nerve regeneration. J Cell Sci 122: 995-1004, 2008.

19. Goetsch SC, Hawke TJ, Gallarde TD, Richardson JA and Garry DJ: Transcriptional profiling and regulation of the extracellular matrix during muscle regeneration. Physiol Genomics 14: 261-271, 2003.

20. Sharma MK, Watson MA, Lyman M, Perry A, Aldape KD, Deak F and Gutmann DH: Matrilin-2 expression distinguishes clinically relevant subset of pilocytic astrocytoma. Neurol 66: 127-130, 2006
21. Zhang Y, Wang Z, Luo J, Kanbe K and Chen Q: Multiple functions of the von Willebrand factor A domain in matrilins: secretion, assembly, and proteolysis. J Orthop Surg Res 3: 21, 2008.

22. Ehlen HW, Sengle G, Klatt AR, Talke A, Muller S and Paulsson M: Proteolytic processing causes extensive heterogeneity of tissue matrilin forms. J Biol Chem 284: 21545-21556, 2009.

23. O'Reilly MS, Holmgren L, Chen C and Folkman J: Angiostatin induces and sustains dormancy of human primary tumors in mice. Nat Med 2: 689-692, 1996.

24. Bergers G, Javaherian K, Lo KM, Folkman J and Hanahan D: Effects of angiogenesis inhibitors on multistage carcinogenesis in mice. Science 284: 808-812, 1999.

25. Krieg T and LeRoy EC: Diseases of the extracellular matrix. J Mol Med 76: 224-225, 1998. 